\title{
PRODUTIVIDADE, SALÁRIOS \\ E TAXA DE CÂMBIO: \\ UMA ANÁLISE DA EXPERIÊNCIA BRASILEIRA NOS ANOS 1990*
}

\author{
Eduiges Romanatto ${ }^{* *}$
}

\section{Gabriel Porcile ${ }^{* * *}$}

\section{Marcelo Curado ${ }^{* * * *}$}

RESUMO O propósito deste trabalho é analisar as relações entre salário real, produtividade, emprego e taxa de câmbio real na economia brasileira durante os anos 1990. Esse período é particularmente interessante, já que combinou liberalização comercial, valorização da moeda e aumentos elevados de produtividade. Com esse objetivo, testou-se, através de um modelo VEC, um modelo teórico baseado em concorrência monopolística. $\mathrm{O}$ modelo fez as previsões corretas quanto à relação salário real e produtividade, mas não quanto à relação salário real e emprego.

Palavras-chave: produtividade; salários; taxa de câmbio; modelos VAR

Código JEL: C22, E24

\section{PRODUCTIVITY, WAGES AND EXCHANGE RATE: AN ANALYSIS OF THE BRAZILIAN EXPERIENCE IN THE NINETIES}

ABSTRACT This paper discusses the interrelations between real wages, the real exchange rate and productivity in the Brazilian economy during the nineties. This

* Artigo recebido em 4 de dezembro de 2007 e aprovado em 30 de setembro de 2008.

** Mestre em Desenvolvimento Econômico pela UFPR, e-mail: tgromanatto@yahoo.com.br

*** Doutor em Economia pela LSE e professor do Departamento de Economia da UFPR, e-mail: porcile@ufpr.br

**** Professor do Departamento de Economia da Universidade Federal do Paraná e doutor em Economia pela Unicamp, e-mail: mcurado@ufpr.br 
period is particularly interesting as it combined trade liberalization, the valorization of the Real and relatively high rates of growth of labor productivity. With this objective, a VEC model was used to test a theoretical model based on monopolisitic competition. The model predicted correctly the association between real wages and productivity, but it did not perform well as regards real wages and unemployment.

Key words: productivity; wages; exchange rate; VAR models 


\section{INTRODUÇÃO}

O debate sobre o comportamento da produtividade e do salário real na década de 1990 foi intenso e, em muitos aspectos, permanece sem resolução. Todavia, a maior parte dos autores parece concordar em dois aspectos:

(i) os ganhos de produtividade foram muito significativos nesse período;

(ii) eles não foram repassados para os salários reais em uma magnitude equivalente.

Em nível setorial, os estudos sobre os vínculos entre produtividade e salários reais não mostram a existência de uma relação significativa entre essas variáveis. No entanto, há evidências para o conjunto da economia de que os salários reais aumentaram nos anos 1990, embora de forma modesta. É possível, então, que os determinantes desse aumento não se encontrem em nível setorial, mas em nível macroeconômico. Em particular, alguns modelos macroeconômicos baseados na concorrência monopolística sugerem que, além da produtividade e emprego, uma taxa de câmbio mais baixa pode - por meio da operação de distintos mecanismos - estar associada a um salário real mais elevado.

Assim, o objetivo deste trabalho é testar as inter-relações entre as variáveis salários reais, desemprego, produtividade e taxa de câmbio real, em nível macroeconômico, baseado na concorrência monopolística. Os modelos disponíveis para a análise dessas relações admitem que essas variáveis são determinadas em um sistema de equações dinâmicas, em que todas as variáveis são endógenas. Para isso, um instrumento adequado é o sistema de Vetores Auto-regressivos (VAR), com as restrições impostas pelas relações de equilíbrio de longo prazo entre as variáveis (equação de co-integração).

Além desta introdução, o presente trabalho está organizado da seguinte forma: a seção 1 apresenta uma breve revisão envolvendo a variável salário real, desemprego e produtividade para a economia brasileira; a seção 2 apresenta uma versão simplificada do modelo macroeconômico com tais variáveis mais a taxa de câmbio; a seção 3 apresenta os dados, a metodologia, os resultados e algumas análises; e a seção 4 faz as considerações finais. 


\section{SALÁRIO REAL, PRODUTIVIDADE E EMPREGO PARA A ECONOMIA BRASILEIRA NA DÉCADA DE 1990}

Com relação ao tema dos ganhos de produtividade a partir de 1996, parece haver um consenso de que existiram aumentos importantes de produtividade na indústria brasileira, como se resume no quadro 1 a seguir. Mesmo autores que, inicialmente, tenderam a questionar essa hipótese (Considera e Valadão, 1995), reconheceram, posteriormente, que esses aumentos foram significativos (Considera, 1996 e 1998). É verdade que provavelmente os aumentos tenham sido sobreestimados, em função dos efeitos negativos da terceirização sobre o emprego. Ainda assim, os anos 1990 foram bastante dinâmicos do ponto de vista do comportamento da produtividade.

A sintonia entre os autores quanto aos ganhos de produtividade não é encontrada quando se discute quem se beneficiou desses aumentos: não há consenso, como se verifica no quadro 2, quanto ao padrão de distribuição da produtividade para o salário. Ao que parece, os benefícios se deram de forma específica e em diferentes magnitudes para cada setor, sem estar relacionados de forma sistemática ao comportamento da produtividade.

Quadro 1: Ganhos de produtividade na economia brasileira

\begin{tabular}{ll}
\hline Autores & Ganhos de produtividade \\
\hline $\begin{array}{l}\text { Silva et al. (1994) } \\
\text { Considera e }\end{array}$ & $\begin{array}{l}\text { Não (devido a ajuste recessivo natural) } \\
\text { Não de forma generalizada, apenas localizada (devido a } \\
\text { ajuste recessivo natural e ao fenômeno da terceirização) }\end{array}$ \\
\hline Coutinho (1997) & Não faz menção \\
\hline Feijó e Carvalho (1999) & Sim (novos processos de produção e de reorganização) \\
\hline Amadeo e Villela (1994) & Sim (reestruturação da indústria brasileira) \\
\hline $\begin{array}{l}\text { Salm, Saboia e Carvalho } \\
\text { (1997) }\end{array}$ & $\begin{array}{l}\text { Sim (novos métodos de gestão da produção e aumento da } \\
\text { competitividade das empresas) }\end{array}$ \\
\hline Bonelli e & $\begin{array}{l}\text { Sim (grande parte do produto potencial seria explicada pela } \\
\text { produtividade) }\end{array}$ \\
\hline Conseca (1998) & Sim (mesmo com os dados contendo sobreestimação) \\
\hline Rossi Jr. e Ferreira (1999) & Sim (mudança estrutural e ganhos generalizados) \\
\hline Bonelli (2002) & Sim (uma inversão em relação à década de 1980) \\
\hline Cavalheiro (2003) & Sim (pequeno aumento e de maneira desigual) \\
\hline Feijó (2003) & Sim (produto cresceu e emprego ficou estagnado) \\
\hline Amadeo e Soares (1996) & Sim (mudança estrutural na série produtividade) \\
\hline Cacciamali e Bezerra (1997) & Sim (mudança estrutural na série produtividade) \\
\hline Fonte: Elaborado pelos autores. & \\
\hline
\end{tabular}


Um dos motivos da falta de consenso pode ser a qualidade dos dados, ${ }^{1}$ assim como a dificuldade de controlar a influência de outros fatores como a estabilização de preços, taxa de câmbio, privatização e desregulamentação de mercados, entre outras políticas adotadas na década de 1990, que podem ter influenciado significativamente o mercado de trabalho e o comportamento dos preços relativos.

Quanto à relação entre ganhos de produtividade e emprego, o debate também permanece aberto, conforme quadro 3. Outras forças contribuíram para a determinação das variações do emprego, já que, conforme Feijó (2003), a produtividade cresceu a taxas médias menores (1,7\% a.a.) que a do PIB (2,5\% a.a), e a expansão do emprego foi fraca na década de 1990 $(0,9 \% \text { a.a. })^{2}$ As transformações que se processaram na estrutura produtiva

\section{Quadro 2: Produtividade e salários}

\begin{tabular}{|c|c|c|c|}
\hline Autores & $\begin{array}{l}\text { Período } \\
\text { de } \\
\text { análise }\end{array}$ & $\begin{array}{l}\text { Produtividade } \\
\text { explica } \\
\text { salários }\end{array}$ & $\begin{array}{l}\text { Variável de } \\
\text { comparação } \\
\text { com produtividade }\end{array}$ \\
\hline Franco (1998) & $\begin{array}{l}\text { Pós-Plano } \\
\text { Real (94-96) }\end{array}$ & Sim & - \\
\hline Chamon (1998) & $90-96$ & Sim & Salário real \\
\hline $\begin{array}{l}\text { Bonelli e } \\
\text { Fonseca (1998) }\end{array}$ & $86-98$ & Sim & $\begin{array}{l}\text { Participação da folha sala- } \\
\text { rial no valor da produção }\end{array}$ \\
\hline $\begin{array}{l}\text { Feijó e Carvalho } \\
\text { (1999) e (2000) }\end{array}$ & $90-97$ & Não & $\begin{array}{l}\text { Participação do salário } \\
\text { no valor agregado }\end{array}$ \\
\hline Bonelli (2002) & $\begin{array}{l}90-00 \\
90-94\end{array}$ & $\begin{array}{l}\text { Não há padrão definido } \\
\text { Sim }\end{array}$ & $\begin{array}{l}\text { Salário real } \\
\text { Salário real }\end{array}$ \\
\hline $\begin{array}{l}\text { Souza Neto e } \\
\text { Curado (2005) } \\
\end{array}$ & $\begin{array}{l}94-00 \\
90-00 \\
\end{array}$ & $\begin{array}{l}\text { Não } \\
\text { Não }\end{array}$ & \\
\hline Arbache e Courseuil (2001) & $87-98$ & $\begin{array}{l}\text { Não para setores exportadores } \\
\text { e sim para setores importadores }\end{array}$ & Dados da PNAD \\
\hline
\end{tabular}

Fonte: Elaborado pelos autores.

Quadro 3: Produtividade e emprego

\begin{tabular}{ll}
\hline Autores & Relação entre produtividade e emprego \\
\hline Villela e Silva (1994) & Não existe relação direta \\
\hline Considera e Valadão (1995) & $\begin{array}{l}\text { Não de forma generalizada, apenas localizada (devido a ajuste } \\
\text { recessivo natural e ao fenômeno da terceirização) }\end{array}$ \\
\hline Ramos e Reis (1997) & Relação pequena e com outras variáveis afetando queda do emprego. \\
\hline Feijó e Carvalho (2000) & Não há padrão de comportamento \\
\hline Bonelli e Fonseca (1998) & Correlação pequena \\
\hline Cavalheiro (2003) & $\begin{array}{l}\text { Mudança na estrutura do trabalho é que atuou na diminuição da } \\
\text { produtividade }\end{array}$ \\
\hline
\end{tabular}


do país, particularmente com a realocação de mão-de-obra da indústria para o setor de serviços, juntamente com o baixo crescimento econômico da década, confluem para explicar o baixo crescimento do emprego.

Finalmente, no que se refere à relação entre emprego e salários, autores como Chamon (1998), Camargo, Neri e Reis (1999) e Arbache e Courseuil (2001) sugerem que a queda no emprego não significou queda nos ganhos salariais na indústria brasileira. A que se deveria essa falta de reação dos salários? A uma situação mais difícil no mercado de trabalho? A liberalização comercial, o aumento da concorrência e a introdução de novas formas de organização do trabalho, juntamente com o comportamento da taxa de câmbio na década de 1990, seriam fatores que poderiam explicar esse fenômeno. Aumentos de produtividade e redução da taxa de câmbio real poderiam ter neutralizado o efeito negativo do desemprego sobre o salário real.

Assim, é necessário considerar esse conjunto de variáveis de forma integrada - desemprego, salários reais, produtividade e taxa de câmbio real - olhando para sua dinâmica no contexto de uma economia em transformação na década de 1990. O estudo empírico realizado, apresentado nas próximas seções, avança precisamente nessa direção.

\section{O MODELO MACROECONÔMICO}

O salário real é visto como um dos mais importantes preços da economia. Ele contribui para definir a distribuição da renda e o nível de emprego. Assim, várias teorias, com diferentes estruturas de mercado, tanto em economia fechada quanto em aberta, discutem a determinação dos salários e, para isso, exploram-se as suas ligações com variáveis como taxa de câmbio real, produtividade e nível de emprego.

O modelo de referência é o de concorrência monopolística de Carlin e Soskice (1990), que parte de uma regra de mark-up para bens produzidos e vendidos internamente e no exterior, sem a participação de insumos importados no processo produtivo:

$$
P=P x=\frac{1}{1-m} \frac{W}{a}
$$

onde $m$ é o mark-up, $W$ os salários e o parâmetro $a$ mede a produtividade do trabalho. 
Mas o nível de preços relevantes para o trabalhador é aquele usado no cálculo da reposição dos salários nominais visando a manter o salário real, ou seja, os salários monetários em termos do índice de preços ao consumidor $\left(W / P_{c}\right)$. Admite-se que os trabalhadores consomem uma cesta composta de bens domésticos e importados. Os que são importados têm um preço $e$ $P^{*}$ e os domésticos $P$, onde $e$ é a taxa de câmbio nominal (reais por dólar), $P$ os preços internos e $P^{\star}$ os preços externos. A participação do consumo de importados será $\phi$. Assim, o deflator de preços do salário real é:

$$
P c=(1-\phi) P+\phi e P^{*}
$$

O salário real em termos de índice de preços ao consumidor será:

$$
w=\frac{W}{P c}
$$

Supondo o salário real como uma função decrescente da taxa de desemprego, tem-se:

$$
W=P c \cdot b(U)
$$

Ou ainda:

$$
\frac{W}{P c}=b(U)=w b
$$

O aumento no desemprego é associado com a queda na demanda de salário real dos trabalhadores como resultado do seu menor poder de barganha. Substituindo o nível de preços domésticos, $P$, no IPC, temos (6):

$$
P c=(1-\phi)\left(\frac{1}{1-m} \frac{W}{a}\right)+\phi e P^{*}
$$

Dividindo ambos os lados por $\frac{1}{(1-m)} \frac{W}{a}$, obtemos a equação 7:

$$
\frac{P c(1-m) a}{W}=(1-\phi)+\phi \frac{e P^{*}}{P}
$$


Finalmente, usando $w=\frac{W}{P c}$ e taxa de câmbio real, $q=\frac{e P^{*}}{P}$, obtemos:

$$
b(U)=w p=\frac{a(1-m)}{1+\phi(q-1)}
$$

Supondo $m$ e $\phi$ constantes, temos que o nível de salário real se relaciona positivamente com a produtividade do trabalho $(a)$ e inversamente com a taxa de câmbio real $(q)$. As previsões do modelo são de que o salário real aumenta quando aumenta a produtividade ou quando cai a taxa de câmbio real. O modelo também mostra que essas variáveis interagem no tempo para determinar salário real e emprego. Nas próximas seções essa interação e as previsões do modelo serão analisadas empiricamente.

\section{DADOS, METODOLOGIA, RESULTADOS E ANÁLISE ${ }^{3}$}

Os dados referentes a emprego e produtividade são obtidos da Pesquisa Industrial Mensal — Produção Física e Dados Gerais (PIM-PF e PIM-DG) ${ }^{4}$ do IBGE. Sobre essas séries, Feijó e Carvalho (1999) apontam que o maior problema é o "envelhecimento" de suas amostras, já que são usados os Censos Industriais da década de 1980 como referência. Mas eles indicam corretamente a tendência das variáveis pesquisadas. Dessa forma, utiliza-se a taxa de desemprego aberto - 30 dias e abrange as regiões metropolitanas, bem como o cálculo da produtividade para este trabalho compreende a divisão da produção física da indústria como um todo pelo número de trabalhadores ocupados nela.

Uma limitação está no cálculo da variável produtividade utilizando-se uma medida de produção (PIM-PF) e uma de insumo trabalho (PIM-DG), que são de amostras diferentes, embora ambas do IBGE. Assim, por exemplo, os coeficientes técnicos provavelmente mudaram na década de 1990 (dada a abertura comercial) e a medida de produção usada como proxy irá gerar resultados viesados. Porém, conforme Bonelli e Fonseca (1998), com a divulgação das contas nacionais, o viés foi “favorável” (ou oposto ao esperado), ou seja, crescimento maior que o da PIM-PF. De outra maneira, se existir viés no insumo trabalho, a série produtividade também resultará vie- 
sada. Contudo, em cuidadosa avaliação desses argumentos, Salm, Saboia e Carvalho (1997) concluem que as estatísticas da PIM-DG parecem mais confiáveis para a mensuração da produtividade. Conclusão semelhante encontra-se em Bonelli (1996). Por isso, dá-se preferência em trabalhar com os dados da PIM-PF e DG do IBGE.

Outro problema está relacionado à mensuração da produtividade da mão-de-obra e tem a ver com a variável utilizada no denominador da relação, isto é, a medida de insumos de mão-de-obra. Bonelli e Fonseca (1998) argumentam que o problema maior é que existe a possibilidade de que a PIM-DG tenha perdido representatividade ao longo do tempo, e isso pode dar um viés excessivamente negativo. Afirmam que esse problema não tem resposta simples; no entanto, a taxa de variação do pessoal ocupado, segundo a PIM-DG, e a do pessoal ocupado com carteira assinada, segundo a PME (IBGE, média das seis regiões metropolitanas pesquisadas), indicam resultados de mesma ordem e grandeza. Isso fornece uma indicação de que, se a amostra da PIM-DG perdeu representatividade, esse processo não foi tão intenso.

Outro aspecto importante na determinação dos ganhos de produtividade é a relação da redução do emprego industrial com o processo de terceirização, que foi visível nos anos 1990. Para Bonelli e Fonseca (1998), dois aspectos justificam um efeito não tão forte. Primeiro, a medida de emprego utilizada nos cálculos de produtividade refere-se ao pessoal ligado à produção, e, como se sabe, o fenômeno da terceirização atingiu mais fortemente as atividades não diretamente ligadas ao processo produtivo.

A tabela 1 apresenta o comportamento das variáveis emprego, produção física e produtividade, em suas séries anuais. Entre outros aspectos, é possível notar pela tabela que boa parte dos incrementos de produtividade encontram-se associados à forte redução do emprego ocorrida na década de 1990.

O salário é a série salário contratual médio real fornecido pelo IBGE. A taxa de câmbio é a efetiva real - INPC - exportações - índice (média 1995 = 100) — fornecida pelo IPEA. ${ }^{5}$ Todos os dados são mensais entre o período de janeiro de 1990 a dezembro de 1998.

A abordagem empírica está baseada na estrutura de um modelo de autoregressão vetorial (VAR) que tem como objetivo a análise da trajetória das variáveis e seus impactos sobre as demais variáveis do modelo. 
Tabela 1: Indústria brasileira - número, índice e variação percentual anual de emprego, produção física e produtividade

\begin{tabular}{|c|c|c|c|c|c|c|}
\hline Anos & Emprego & $\begin{array}{l}\text { Produção } \\
\text { física }\end{array}$ & Produtividade & Emprego & $\begin{array}{l}\text { Produção } \\
\text { física }\end{array}$ & Produtividade \\
\hline 1990 & 100,00 & 100,00 & 100,00 & & & \\
\hline 1991 & 90,95 & 97,2 & 106,89 & $-9,0 \%$ & $-2,8 \%$ & $6,9 \%$ \\
\hline 1992 & 84,0 & 93,8 & 111,66 & $-7,6 \%$ & $-3,5 \%$ & $4,5 \%$ \\
\hline 1993 & 82,4 & 101,0 & 122,51 & $-1,9 \%$ & $7,7 \%$ & $9,7 \%$ \\
\hline 1994 & 80,6 & 108,8 & 135,00 & $-2,2 \%$ & $7,7 \%$ & $10,2 \%$ \\
\hline 1995 & 79,1 & 110,9 & 140,26 & $-1,9 \%$ & $1,9 \%$ & $3,9 \%$ \\
\hline 1996 & 70,2 & 112,0 & 159,43 & $-11,2 \%$ & $1,0 \%$ & $13,7 \%$ \\
\hline 1997 & 66,2 & 116,7 & 176,32 & $-5,8 \%$ & $4,2 \%$ & $10,6 \%$ \\
\hline 1998 & 60,1 & 114,4 & 190,21 & $-9,1 \%$ & $-2,0 \%$ & $7,9 \%$ \\
\hline
\end{tabular}

Fonte: Elaboração dos autores com base nas pesquisas industriais mensais do IBGE.

Tabela 2: Testes de raiz unitária de Dickey-Fuller ampliado (ADF) no nível de $5 \%$ de significância

\begin{tabular}{ccccc}
\hline $\begin{array}{c}\text { Variável 1990:01 } \\
\text { a 1998:12 }\end{array}$ & ADF $\left(\tau_{\tau}\right)-3,45$ & Com constante & Com tendência & $\begin{array}{c}\text { Ordem de } \\
\text { integração } I(d)\end{array}$ \\
\hline$a$ & $-3,21(5)$ & $\operatorname{sim}$ & $\operatorname{sim}$ & $\mathrm{I}(1)$ \\
\hline$U$ & $-3,43(3)$ & $\operatorname{sim}$ & $\operatorname{sim}$ & $\mathrm{I}(1)$ \\
\hline$q$ & $-2,50(7)$ & $\operatorname{sim}$ & não & I (1) \\
\hline$w r$ & $-2,29(3)$ & $\operatorname{sim}$ & $\operatorname{sim}$ & $\mathrm{I}(1)$ \\
\hline$\Delta a$ & $-4,89(5)$ & não & são & I (0) \\
\hline$\Delta q$ & $-4,81(4)$ & não & não & I (0) \\
\hline$\Delta u$ & $-7,06(3)$ & não & não & I (0) \\
\hline$\Delta w r$ & $-8,34(3)$ & sim & não & I (0)
\end{tabular}

Fonte: Dados da pesquisa; $(\Delta)$ representa a série em primeira diferença.

Nota: Os dados entre parênteses representam as defasagens do modelo ADF. A variável $q$ é a taxa de câmbio real, $U$ é a taxa de desemprego e wr o salário real.

O primeiro passo no tratamento dos dados é verificar se as séries são ou não estacionárias. Se uma série apresenta raiz unitária, qualquer choque temporário torna-se permanente, ou seja, as flutuações não apresentariam comportamento transitório e a série não convergiria para seu nível de longo prazo. Variáveis não estacionárias geram regressões espúrias quando usadas em níveis e, além disso, as estatísticas usuais como $R^{2}, D W$ e $t$ de Student não mantêm suas características usuais. Pela abordagem Box-Jenkins, uma série não estacionária pode se tornar estacionária por diferenciação. O número de diferenciação determina a ordem de integração da série. Os resultados para os testes de raiz unitária estão na tabela 2 e foram obtidos a partir do estabelecimento da melhor especificação das defasagens via AIC e SBC. ${ }^{6}$ Es- 
Tabela 3: Teste de co-integração de Johansen? para taxa desemprego, taxa de câmbio, produtividade e salário real

\begin{tabular}{|c|c|c|c|c|}
\hline Eigenvalue & $\begin{array}{l}\text { Likelihood } \\
\text { ratio }\end{array}$ & $\begin{array}{c}5 \text { Percent critical } \\
\text { value }\end{array}$ & $\begin{array}{c}1 \text { Percent critical } \\
\text { value }\end{array}$ & $\begin{array}{c}\text { Hypothesized No. } \\
\text { of CE(s) }\end{array}$ \\
\hline 0,203572 & 49,68590 & 47,21 & 54,46 & None* \\
\hline 0,147235 & 26,24122 & 29,68 & 35,65 & At most 1 \\
\hline 0,067004 & 9,836329 & 15,41 & 20,04 & At most 2 \\
\hline 0,025805 & 2,692836 & 3,76 & 6,65 & At most 3 \\
\hline
\end{tabular}

colheu-se aquele modelo com menores AIC e SBC e se aplicou nelas estatísticas ADF no nível de $95 \%$ de probabilidade.

Observa-se na tabela que todas as variáveis em nível apresentam raiz unitária, isto é, aceita-se a hipótese nula de não-estacionariedade. Isso significa que as variáveis não estacionárias devem ser integradas por meio de diferenças e que, também, dever ser novamente testada a ordem de integração. Os resultados das variáveis em primeira diferença, representadas pelos deltas $(\Delta)$, indicam serem todas estacionárias. Os valores das estatísticas ADF foram todos, em módulo, maior do que os valores críticos no nível de $5 \%$ de significância.

O segundo passo é testar a estacionariedade do sistema como um todo. Para isso utiliza-se da metodologia de Johansen. O resultado para as variáveis em questão está na tabela 3.

Os resultados da tabela 3 foram obtidos depois de usar diferentes defasagens até se escolher o seu menor número (quatro defasagens), levando-se em conta a significância dos parâmetros Percebe-se que há co-integração entre as variáveis em conjunto, ou seja, ocorre uma combinação linear que deve ser incluída no VAR. ${ }^{8}$ Se há co-integração no sistema, deve-se fazer a estimação VAR em nível, mas com a inclusão da equação de co-integração ou a utilização de um Vetor de Correção de Erro (VEC). A equação de cointegração resultante foi:

$$
\log w r=14+0,36 \log u-1,1 \log q+0,6 \log a
$$

Pela equação de co-integração, temos uma relação de longo prazo positiva entre salário e desemprego e salário e produtividade. Verifica-se também a existência de uma relação negativa entre salário e taxa de câmbio. 
À primeira vista parece estranho uma relação positiva entre salário real e desemprego, mas, como colocado por Chamon (1998), na década de 1990, ou pelo menos até 1996, ocorreram ganhos nos salários dos trabalhadores, muito embora tenha ocorrido tendência crescente no desemprego. Nessa mesma direção argumentam Camargo, Neri e Reis (1999). A relação positiva entre salários e desemprego pode ser explicada pela terceirização de muitas atividades, que acabou por transferir massa de salários para o setor de serviços, abrindo espaço para aumento dos salários e redução do emprego na indústria.

Já a relação positiva entre salário e produtividade, para o período, estaria de acordo com o argumentado por Chamon (1998) e Bonelli e Fonseca (1998). Ainda contribuiria para isso o aumento das importações, que exerceu grande efeito nas indústrias que empregam trabalho mais qualificado, exigência de novos processos produtivos. Os ganhos de produtividade foram, até certo ponto, repassados para os salários, como indicado por Arbache e Courseuil (2001). Assim, dadas as variáveis de controle utilizadas, tem-se a indicação de que os salários respondem à produtividade em um prazo mais longo.

E, por último, a valorização da taxa de câmbio parece ter influenciado os ganhos salariais, como sugerido por Carlin e Soskice (1990). O câmbio favorável às importações permitiu a introdução de novas tecnologias, aumentando a produtividade, juntamente com a exigência de trabalho mais qualificado para a operação das novas tecnologias, o que resultou em melhores remunerações. Há de se considerar, também, que o controle da inflação, possibilitado em parte por uma taxa de câmbio mais baixa, favoreceu o salário real.

Os impactos da taxa de câmbio real sobre o mercado de trabalho são ainda pouco discutidos pela literatura empírica brasileira. As evidências empíricas disponíveis para a economia norte-americana tendem a convergir para a existência de uma forte relação entre a taxa de câmbio real e os salários e para resultados inconclusivos no que se refere à relação entre essa taxa e o nível de emprego.

Revenga (1992), por exemplo, utilizando dados da economia norte-americana para os anos 1980, encontra forte correlação entre o comportamento da taxa de câmbio e o comportamento dos salários reais e o nível de empre- 
go. Resultados similares são encontrados por Burgess e Knetter (1996). Campa e Goldberg (1998), por outro lado, encontram correlação apenas entre as variáveis taxa de câmbio real e salários reais. A relação entre câmbio e emprego não é encontrada neste trabalho.

Por fim, vale mencionar os resultados encontrados por Goldberg e Tracy (1999). Em um estudo em nível setorial para a economia norte-americana, os autores encontram uma forte correlação entre o comportamento dos salários industriais e os movimentos do dólar norte-americano. Os resultados, no que se refere aos impactos sobre o emprego, são menos evidentes. Dos 20 setores analisados, encontra-se relação entre os movimentos da taxa de câmbio e o emprego em 12 deles.

Uma vez analisada a relação de longo prazo entre as variáveis, o próximo passo é discutir como elas se ajustam no curto prazo. Cada variável pode ser influenciada pelos efeitos retardados de todas as variáveis do modelo. Como há co-integração entre as variáveis em conjunto, ou seja, ocorre uma combinação linear que deve ser incluída no VAR, deve-se fazer a estimação do VAR em nível, mas com a inclusão da equação de co-integração ou a utilização de um Vetor de Correção de Erros (VEC).

O modelo VAR convencional, na forma de médias móveis, exige que a matriz de variância/co-variância dos resíduos seja diagonal, ou seja, permita que o choque de apenas uma variável seja observado. Mas nem sempre essa exigência é atendida e, por conseqüência o choque pode afetar mais de uma variável. Assim, um artifício usado para garantir a diagonalização da matriz de variância/co-variância é a decomposição de Cholesky. Na forma geral, o VAR em questão é dado por:

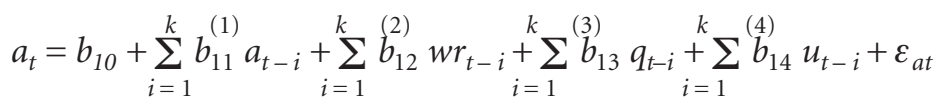

A estrutura do sistema indica relação simultânea entre produtividade (a), salários reais ( $w r)$, taxa de câmbio real $(q)$ e desemprego $(u)$. Supõe-se que as séries tenham as propriedades do modelo Box-Jenkis, ou seja, sejam estáveis, inversíveis, bem como os $\varepsilon_{i t}$ sejam ruído branco com variâncias constantes e não correlacionadas ao longo do tempo.

Um VAR não restringido não assume a presença de co-integração. Para impor a restrição de co-integração entre as variáveis no VAR, usa-se um 
Vetor de Correção de Erros (VCE). Como essas variáveis são co-integradas e determinam uma equação de co-integração, o VAR deve incluir a variável que mede o desvio em relação ao equilíbrio de longo prazo. O VCE permite que os componentes de longo prazo obedeçam às restrições de equilíbrio, enquanto os componentes de curto prazo tenham comportamento dinâmico ou flexível.

Aplicou-se o critério de AIC e SBC, ${ }^{9}$ ou seja, escolhendo-se o número de defasagens que proporciona o menor valor dessas estatísticas. Depois disso, utilizou-se a função de impulso resposta para analisar as inter-relações entre as variáveis. A ordenação destas não alterou o padrão dos resultados.

A estimação do VAR, seguindo os passos descritos anteriormente, proporciona os resultados de decomposição da variância e função de impulso resposta que são apresentados nas figuras 1 e 2 e nas tabelas 4, 5, 6 e 7 .

No gráfico de resposta a impulsos mostra-se o efeito de um choque em uma variável levando-se em conta os efeitos simultâneos de todas as variáveis incluídas no sistema. Relacionou-se, nesses gráficos, o efeito contemporâneo completo de cada variável sobre os efeitos contemporâneos das outras variáveis. Tanto a função de impulso resposta (FRI) quanto a decomposição da variância são relações de curto prazo.

Na decomposição da variância percebe-se que, em geral, as variáveis foram afetadas principalmente pelo seu próprio comportamento. $\mathrm{O}$ mesmo acontece com a resposta a impulsos, ou seja, verifica-se que os efeitos dos choques sobre as variáveis foram mais significativos sobre a própria variável a quem foi atribuído o choque. Porém, alguns resultados parecem se destacar.

Quanto ao salário real, a decomposição da sua variância mostra uma intensidade crescente da explicação via taxa de câmbio real, alcançando 39\% nos períodos iniciais e 31\% nos períodos subseqüentes. Além disso, a FRI indica que um choque positivo na taxa de câmbio real tende a diminuir os salários reais. Isso está, em princípio, de acordo com o proposto por Carlin e Soskice (1990), ou seja, apreciações de câmbio estão associadas a ganhos de salário real. Confirma-se, também, a perspectiva colocada por alguns autores de que o salário real teve um comportamento ascendente durante os anos 1990. Por exemplo, Chamon (1998) afirma que os salários reais cresceram até 1996, enquanto Camargo, Neri e Reis (1999) sugerem que houve 
Figura 1: Respostas a impulsos nas variáveis taxa de desemprego, taxa de câmbio real, produtividade e salário real

\section{Response to one S. D. innovations}

Response of LOGWR to LOGWR

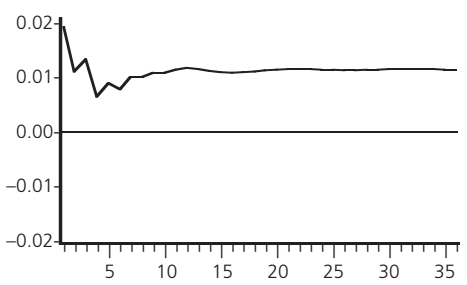

Response of LOGU to LOGWR

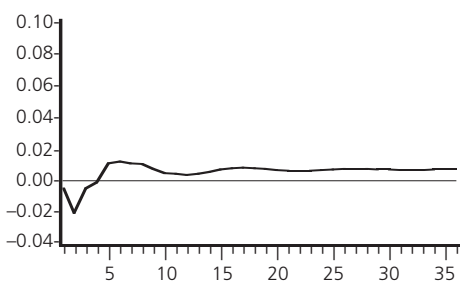

Response of LOGQ to LOGWR

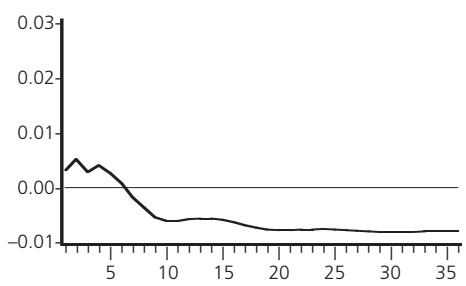

Response of LOGA to LOGWR

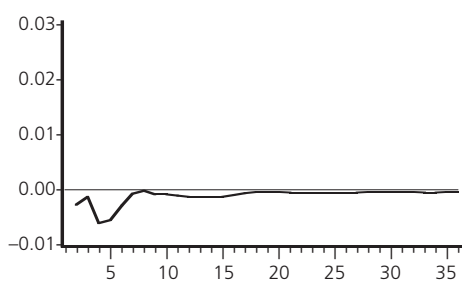

Response of LOGWR to LOGU

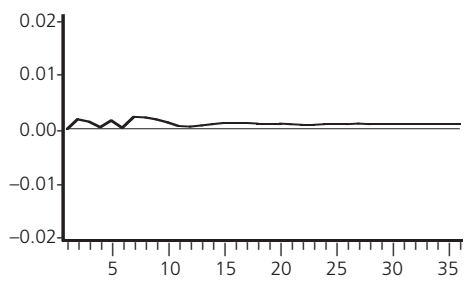

Response of LOGU to LOGU

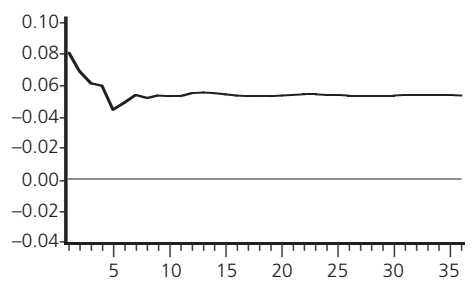

Response of LOGO to LOGU

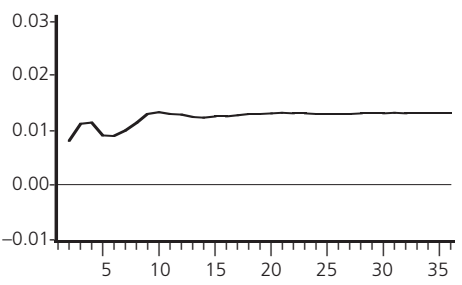

Response of LOGA to LOGU

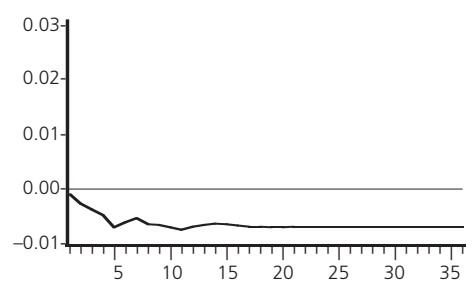




\section{Continuação da figura 1}

Response of LOGWR to LOGQ

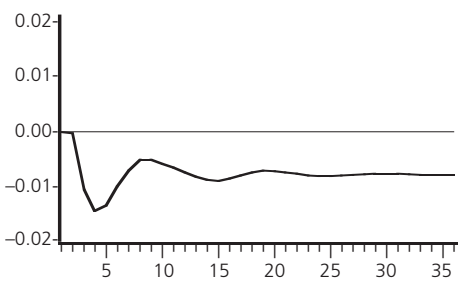

Response of LOGU to LOGQ

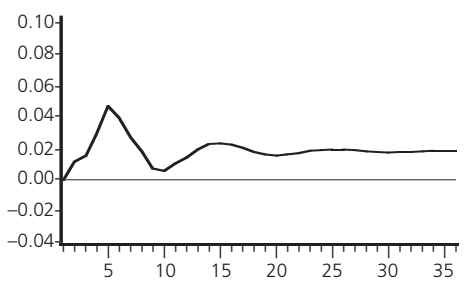

Response of LOGQ to LOGQ

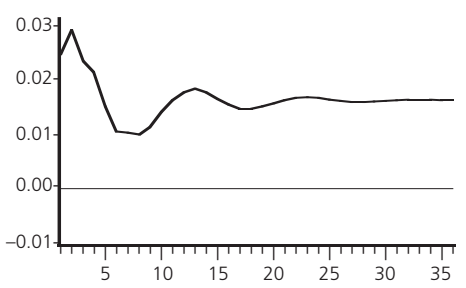

Response of LOGA to LOGQ

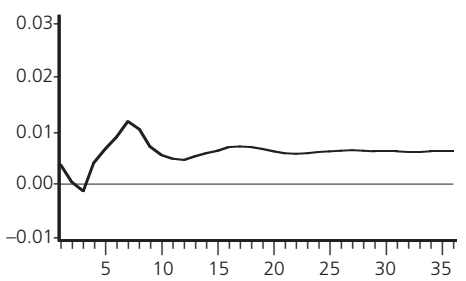

Response of LOGWR to LOGA

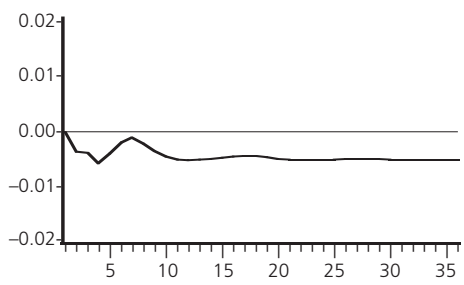

Response of LOGU to LOGA

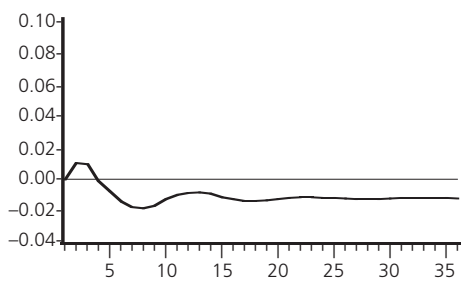

Response of LOGQ to LOGA

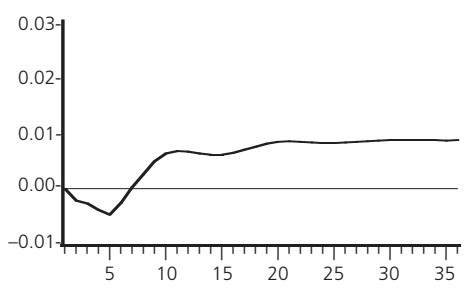

Response of LOGA to LOGA

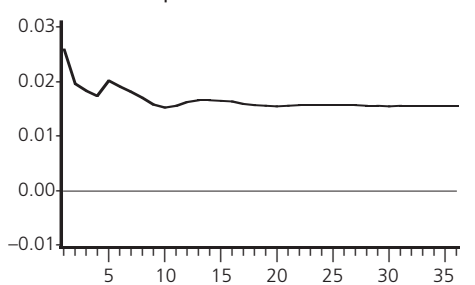


Figura 2: Decomposição da variância

\section{Variance Decomposition}

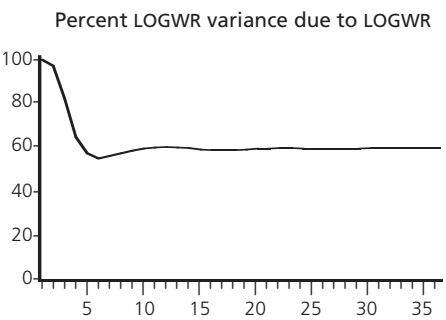

Percent LOGWR variance duo to LOGU

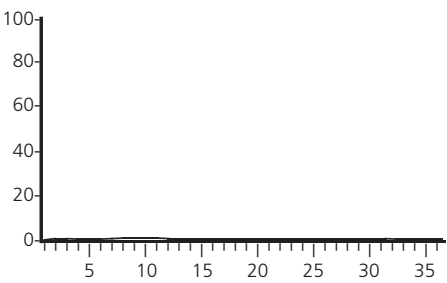

Percent LOGU variance due to LOGWR

Percent LOGU variance due to LOGU
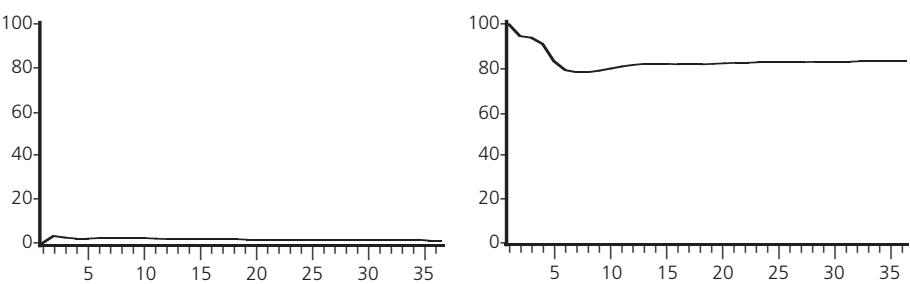

Percent LOGQ variance due to LOGWR

Percent LOGQ variance due to LOGU
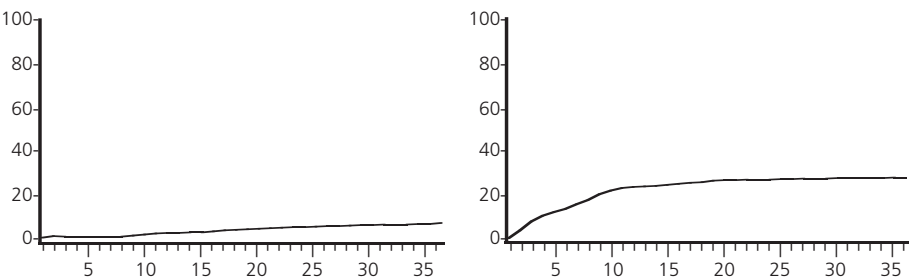

Percent LOGA variance due to LOGWR

Percent LOGA variance due to LOGU
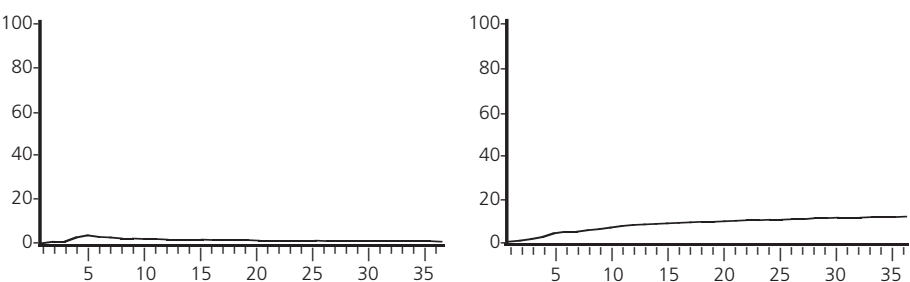


\section{Continuação da figura 2}

Percent LOGWR variance duo to LOGQ

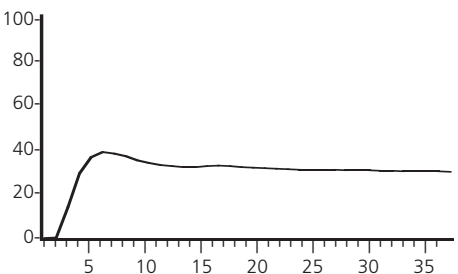

Percent LOGU variance duo to LOGQ

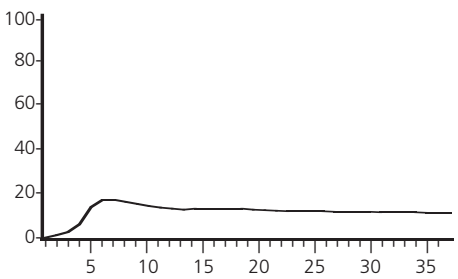

Percent LOGQ variance duo to LOGQ

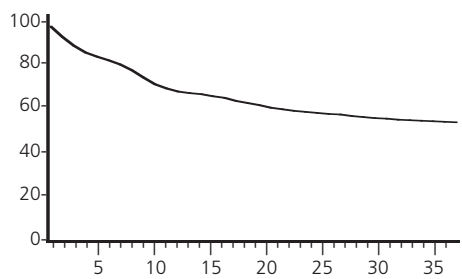

Percent LOGA variance duo to LOGQ

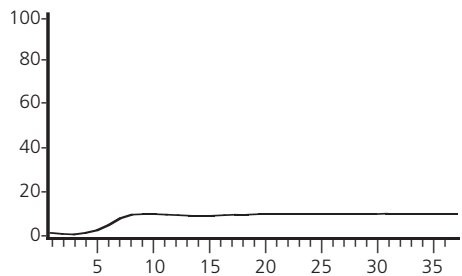

Percent LOGWR variance duo to LOGA

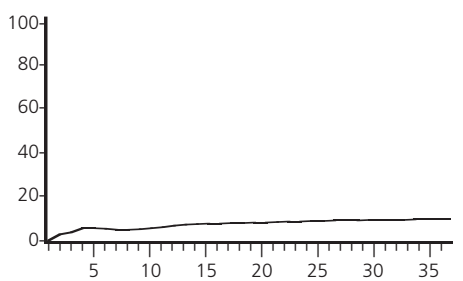

Percent LOGU variance duo to LOGA

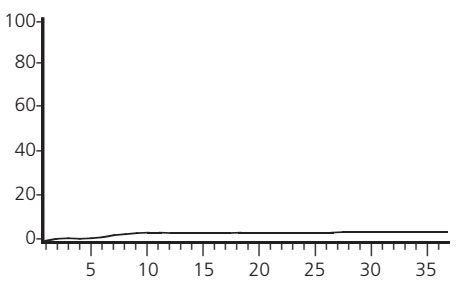

Percent LOGQ variance duo to LOGA

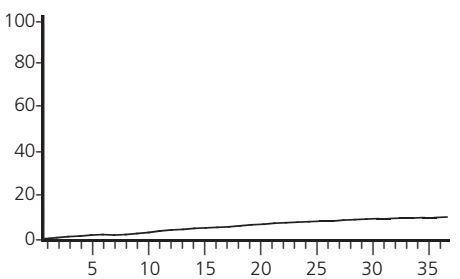

Percent LOGA variance duo to LOGA

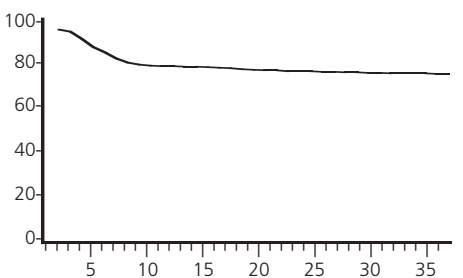


Tabela 4: Decomposição da variância do salário real (LOGWR)

\begin{tabular}{cccccc}
\hline Period & S.E. & LOGWR & LOGU & LOGQ & LOGA \\
\hline 1 & 0.019175 & 100.0000 & 0.000000 & 0.000000 & 0.000000 \\
\hline 6 & 0.039183 & 54.94228 & 0.436245 & 39.27321 & 5.348265 \\
\hline 12 & 0.050670 & 59.79678 & 0.750646 & 32.58472 & 6.867856 \\
\hline 20 & 0.065240 & 59.16046 & 0.617109 & 31.85203 & 8.370397 \\
\hline 24 & 0.071608 & 59.24683 & 0.561417 & 31.19395 & 8.997805 \\
\hline
\end{tabular}

Fonte: Resultados da análise empírica.

Tabela 5: Decomposição da variância do desemprego (U)

\begin{tabular}{cccccc}
\hline Period & S.E. & LOGWR & LOGU & LOGQ & LOGA \\
\hline 1 & 0.080636 & 0.342406 & 99.65759 & 0.000000 & 0.000000 \\
\hline 6 & 0.170566 & 2.572230 & 78.66800 & 17.19196 & 1.567813 \\
\hline 12 & 0.221995 & 2.289904 & 81.13089 & 13.06707 & 3.512141 \\
\hline 18 & 0.265749 & 2.042190 & 81.36086 & 12.98033 & 3.616625 \\
\hline 24 & 0.301475 & 1.889791 & 82.31604 & 12.02696 & 3.767210 \\
\hline
\end{tabular}

Fonte: Resultados da análise empírica.

crescimento do salário real entre 1994 e 1998. Como esse período coincide com o do Plano Real, parece plausível a idéia de Carlin e Soskice (1990) de que os salários tenham obtido ganhos juntamente com a vigência de uma taxa de câmbio mais baixa.

Os salários parecem não ter influência contemporânea significativa do desemprego, tanto pela análise da decomposição quanto pela função de impulso resposta (FRI). Isso estaria na direção do argumentado por Chamon (1998), Camargo, Neri e Reis (1999) e Arbache e Courseuil (2001), em que outros fatores afetaram os salários. Apesar de ter ocorrido uma relação positiva de longo prazo através da equação de co-integração entre aumentos nos ganhos salariais e aumento do desemprego, parece que não há efeitos de curto prazo.

A relação de curto prazo entre salário e produtividade é pouco significativa, cerca de $8 \%$ da sua variância. Já a FRI indica uma influência negativa da produtividade nos salários, contrariando o esperado. Isso pode estar associado ao fato de que a FRI são influências de curto prazo, ou ainda devido à periodicidade dos dados, que são mensais. Acrescenta-se a isso o fato de que as negociações salariais são feitas em intervalos maiores do que o mensal, o que implica uma rigidez no repasse da produtividade para salários, então 
Tabela 6: Decomposição da variância da taxa de câmbio (Q)

\begin{tabular}{cccccc}
\hline Period & S.E. & LOGWR & LOGU & LOGQ & LOGA \\
\hline 1 & 0.024834 & 1.768871 & 0.768879 & 97.46225 & 0.000000 \\
\hline 6 & 0.058201 & 2.096012 & 14.38287 & 81.93627 & 1.584850 \\
\hline 12 & 0.075532 & 3.867220 & 24.29787 & 67.90985 & 3.925056 \\
\hline 19 & 0.096196 & 5.507204 & 27.00599 & 61.36403 & 6.122779 \\
\hline 22 & 0.104666 & 6.267485 & 27.52898 & 58.99851 & 7.205019 \\
\hline 25 & 0.112546 & 6.768318 & 27.81109 & 57.50004 & 7.920549 \\
\hline
\end{tabular}

Fonte: Resultados da análise empírica.

Tabela 7: Decomposição da variância da produtividade (A)

\begin{tabular}{cccccc}
\hline Period & S.E. & LOGWR & LOGU & LOGQ & LOGA \\
\hline 1 & 0.026100 & $4.60 \mathrm{E}-05$ & 0.135458 & 1.752207 & 98.11229 \\
\hline 6 & 0.053051 & 3.012514 & 4.648452 & 5.125298 & 87.21374 \\
\hline 12 & 0.070924 & 1.773325 & 7.801463 & 9.750955 & 80.67426 \\
\hline 18 & 0.084407 & 1.333572 & 9.174330 & 10.15166 & 79.34044 \\
\hline 24 & 0.095239 & 1.061667 & 10.30051 & 10.24146 & 78.39636 \\
\hline
\end{tabular}

Fonte: Resultados da análise empírica.

existem razões para pensar que a relação de longo prazo demora a ser observada. Mais ainda, a decomposição da variância indica um efeito muito pequeno da produtividade sobre o salário real. De toda forma, a relação negativa no curto prazo, embora pequena, permanece como um paradoxo em termos dos resultados esperados pelo modelo.

O segundo resultado é que o desemprego não é influenciado significativamente, no curto prazo, tanto na decomposição da variância quanto na FRI, pelos salários e pela produtividade. A indicação de Bonelli e Fonseca (1998) é a de que não há relação do desemprego com a produtividade, mas, conforme Feijó e Carvalho (2000), essa questão está, em parte, ainda em aberto. Os resultados aqui estariam na direção dos primeiros.

Temos ainda que o desemprego guarda relação, apesar de pequena, com a taxa de câmbio real, cerca de $12 \%$ da sua variância. Pela FRI, vê-se que essa relação é positiva — aumentos na taxa de câmbio nos anos 1990 estiveram associados a um aumento do desemprego no curto prazo. A relação positiva entre o aumento da taxa de câmbio e o aumento do desemprego pode estar associada ao fato de que no curto prazo a desvalorização não gerou uma resposta nas exportações e na demanda efetiva. Mais provavelmente, ela teve um efeito recessivo, seja porque aumentou a incerteza a respeito da política 
cambial, reduzindo o investimento, seja porque afetou negativamente o salário real, reduzindo o consumo. Também pode ser explicada pela crise ocorrida no início da década (1990-1991), em que o desemprego aumentou juntamente com elevações da taxa de câmbio, bem como pela estabilização e valorização da moeda ocorrida com o Plano Real, em que, de início, houve um aquecimento da economia aumentando o emprego. Depois de meados de 1995, o câmbio voltou a ter trajetória crescente juntamente com o desemprego.

Como terceiro resultado, temos a influência das distintas variáveis sobre a taxa de câmbio real: o desemprego (cerca de $27 \%$ da sua variância), a produtividade (7\%) e o salário real (6\%). É interessante notar que um choque positivo no desemprego produz o efeito de depreciar a taxa de câmbio. Como uma hipótese preliminar, poder-se-ia argumentar que essa relação deriva do efeito do desemprego sobre as expectativas da economia; isto é, o aumento de desemprego, juntamente com pouco crescimento do produto, como ocorreu nos anos 1990, gerou expectativas negativas sobre a viabilidade das políticas, o que por sua vez se expressa em uma elevação do câmbio.

Por último, a produtividade parece não guardar relação com os salários, mas parece ter certa influência da taxa de câmbio e do desemprego (10\% para ambas as variáveis). Pela FRI, o aumento do desemprego parece aumentar a produtividade. Apesar de pequeno, o efeito do desemprego sobre a produtividade é maior do que o da produtividade sobre o desemprego, como visto anteriormente.

\section{CONSIDERAÇÕES FINAIS}

A indústria brasileira nos anos 1990 mostrou um crescimento da produtividade do trabalho e dos salários. $\mathrm{O}$ crescimento da produtividade parece indicar que mudanças estruturais aconteceram na economia nesse período, como fruto da redefinição da sua inserção no cenário internacional. Todavia, os aumentos de produtividade não se refletiram, em nível setorial, em aumentos dos salários reais, embora uma relação positiva seja observada para o conjunto da economia. Outros fatores parecem ter desempenhado um papel chave na evolução dessas variáveis. Em particular, no mesmo período, aumentou o desemprego, e a taxa de câmbio real permaneceu bastante valorizada, o que sugere a necessidade de analisar o ambiente macroeconômico. 
Como esperado pelo modelo baseado na concorrência monopolística, o salário real tem uma relação de longo prazo positiva com a produtividade e negativa com a taxa de câmbio real. No entanto, o salário real e o desemprego estiveram positivamente associados, um resultado previsto pelos modelos baseados em concorrência perfeita. Este último resultado pode ser explicado pela terceirização de muitas atividades, que acabou por transferir a massa de salários para o setor de serviços, abrindo espaço para o aumento dos salários e a redução do emprego na indústria. Outra explicação plausível é que, ao menos no curto prazo, é possível verificar aumento do salário e do desemprego, tendo em vista as características do mercado de trabalho brasileiro. Como as demissões tendem a atingir com maior intensidade os trabalhadores que têm menor escolaridade e remuneração, pode-se obter, por efeito composição, uma elevação do salário na média.

A relação positiva de longo prazo entre salários e produtividade está na direção esperada pela teoria econômica e já destacada por alguns autores na literatura sobre a indústria brasileira nos anos 1990, a saber, de que os ganhos salariais foram positivamente influenciados pela produtividade.

Os ajustamentos de curto prazo das variáveis, através da metodologia VAR, apontam, de maneira geral, para um papel chave do choque da própria variável na decomposição da variância e na função de impulso resposta. Destaca-se a relação negativa entre salário e câmbio, o que está na mesma direção da relação de longo prazo apresentada retro, e uma relação direta entre desemprego e a taxa de câmbio real.

Quanto a este último ponto, a relação pode ser explicada pela crise ocorrida no início da década (1990-1991), em que o desemprego aumentou juntamente com elevações da taxa de câmbio, bem como pela estabilização e valorização da moeda ocorrida com o Plano Real. Com efeito, de início, houve um aquecimento da economia aumentando o emprego, mas depois de meados de 1995 o câmbio voltou a ter trajetória crescente, juntamente com o desemprego. Por outro lado, essa relação pode estar associada ao fato de que, no curto prazo, a desvalorização não gerou uma resposta nas exportações e na demanda efetiva. Mais provavelmente, ela teve um efeito recessivo, seja porque aumentou a incerteza a respeito da política cambial, reduzindo o investimento, seja porque afetou negativamente o salário real, reduzindo o consumo. Ou seja, parece que, no curto prazo, o choque na taxa de câmbio real tem um efeito recessivo ao invés de positivo sobre o emprego. 
Cabe destacar, ainda, que produtividade e salários mostram uma relação negativa no curto prazo, apesar de que sua significância é pequena em termos de decomposição da variância. Isso pode estar associado à periodicidade dos dados, que são mensais. Adiciona-se a isso o fato de que as negociações salariais são feitas em intervalos maiores do que o mensal, o que implica uma rigidez no repasse da produtividade para salários; então existem razões para se pensar que a relação de longo prazo demora a ser observada. De toda forma, a relação negativa no curto prazo permanece como um paradoxo em termos dos resultados esperados pelo modelo.

O desemprego parece não sofrer influência significativa dos salários e da produtividade, embora, pela função de impulso resposta, percebe-se que os aumentos de salários tendem a diminuir o desemprego de início, mas depois a elevá-lo. No que tem a ver com a relação entre produtividade e emprego, a função de impulso resposta parece indicar que aumentos de produtividade podem ocorrer com manutenção ou aumento do emprego - um ponto que a literatura em nível setorial também tinha observado.

Uma aproximação macroeconômica ao problema do salário real no Brasil nos anos 1990 permite captar o quadro mais complexo, em que as distintas variáveis se determinam, dadas as importantes mudanças que ocorreram nesse período - abertura comercial, valorização do câmbio e estabilização da inflação. Os modelos baseados na concorrência monopolística, em conjunto com a restrição externa, parecem um caminho promissor para ajudar a esclarecer os distintos elementos que se conjugaram para determinar a evolução do salário real.

\section{NOTAS}

1. Rocha (2007) realiza uma importante discussão sobre a qualidade dos dados utilizados em trabalhos empíricos da área, com ênfase especial nos problemas existentes no emprego da produtividade física. No entanto, ainda que os argumentos do autor sejam pertinentes, deve-se levar em consideração que a literatura econômica brasileira sobre o tema, em sua extensa maioria, utiliza a medida física da produtividade.

2. A autora usa dados das contas nacionais $(\mathrm{CN})$.

3. Os resultados são obtidos usando-se o pacote econométrico Eviews 4.0.

4. A série PIM-DG foi reformulada a partir de dezembro de 2001. Desde então a pesquisa mudou de nome, passando a se chamar Pesquisa Mensal de Emprego e Salário (PIMES). 
5. Deve-se ressalvar que a taxa de desemprego se refere a regiões metropolitanas, e o emprego, a toda a indústria, localizada ou não em regiões metropolitanas.

6. Akaike Information Criterion (AIC) e Schwartz Bayesian Criterion (SBC).

7. Para uma discussão aprofundada sobre o teste, ver Johansen (1995).

8. Para uma discussão sobre os aspectos metodológicos dos modelos VAR, ver, entre outros, Stock e Watson (2001).

9. Para uma revisão geral desses e de outros critérios de informação, ver, por exemplo, Priestley (1981), cap. 5.

\section{REFERÊNCIAS BIBLIOGRÁFICAS}

AMADEO, E. J.; SOARES, R. R. Quebra estrutural da relação entre produção e emprego na indústria brasileira. Texto para Discussão, Rio de Janeiro: Departamento de Economia PUC-Rio, n. 356, jul. 1996.

; VILLELA, A. Crescimento da produtividade e geração de empregos na indústria brasileira. Revista do BNDES, Rio de Janeiro, v. 1, n.1, p. 51-70, jun. 1994.

ARBACHE, T. S.; COURSEUIL, C. H. Liberalização comercial e estruturas de emprego e salário, IPEA. Texto para Discussão, Rio de Janeiro, n. 801, 2001.

BARRO, R. J. Macroeconomics. Nova York: Wiley, 1990.

BONELLI R. Growth and productivity in brazilian industries. Journal of Development, Northolland, n. 39, 1992.

Labor productivity in Brazil during the 1990s. Texto para Discussão, Rio de Janeiro: IPEA, n. 906, set. 2002.

Produtividade industrial nos anos 90: controvérsia e quase-fatos. A economia brasileira em perspectiva. Rio de Janeiro: IPEA, 1996.

BONELLI, R.; FONSECA, R. Ganhos de produtividade e de eficiência: novos resultados para a economia brasileira. Pesquisa e Planejamento Econômico, Rio de Janeiro, v. 28, n.2, p. 273 314, ago. 1998.

BRAGA, H.; ROSSI, J. Produtividade total do fatores de produção na indústria brasileira: 197083. Pesquisa e Planejamento Econômico, Rio de Janeiro, v. 19, n. 2, p. 255-263, 1989.

BURGESS, S.; KNETTER, M. Na international comparison of employment adjustment of exchange rate fluctuations. NBER Working Paper, n. 5.861, 1996.

CACCIAMALI, M. C.; BEZERRA, L. L. Produtividade e emprego industrial no Brasil, Revista Brasileira de Economia, Rio de Janeiro, v. 51, n. 1, p. 77-91. jan./mar. 1997.

CAMARGO, J. M.; NERI, M.; REIS, M. C. Emprego e produtividade no Brasil na década de noventa. Texto para Discussão, Departamento de Economia PUC-Rio, n. 405, out. 1999.

CAMPA, J. M.; GOLDBERG, L. S. Employment versus wage adjustment and the US Dollar. NBER Working Paper, n. 6.749, 1998.

- Employment versus wage adjustament and the U.S. dollar. The Review of Economics and Statistics, v. 83, n. 3, p. 477, ago. 2001. 
CARLIN, W.; SOSKICE, D. Macroeconomics and the wage bargain: a modern approach to employment, inflation and the exchange rate. [s.l.]: Oxford University Press, 1990.

CAVALHEIRO, N. Uma decomposição do aumento da produtividade do trabalho no Brasil durante os anos 90. Revista Economia Contemporânea, Rio de Janeiro, p. 81-109, jan./jun. 2003.

CHAHAD, J. P. Z.; LUQUE, C. A. Ajuste no emprego e produtividade na indústria brasileira nos ciclos recessivos da década de oitenta. Análise Econômica, Porto Alegre, p. 21-34, n.17, mar. 1992.

CHAMON, M. Rising wages and declining employement: the braslian manufacturing sector in the 90's. Texto para Discussão, Rio de Janeiro: IPEA, n. 552, mar. 1998.

CHRISTOFIDES, L. N.; OSWALD, A J. Real wages determination and rent-sharing in collective bargaing agreements. Quarterly Journal of Economics, Massachusetts, v. 107, n. 3, p. 9851.002, ago. 1992.

CONSIDERA, C. M. Globalização, produtividade e emprego Industriais. Boletim Conjuntural, IPEA, n. 35, out. 1996.

. Produto, emprego e produtividade industriais: o que se pode aprender das novas contas nacionais? Mercado de Trabalho, Conjuntura e Análise. IPEA, n. 7, p. 25-32, fev. 1998.

; VALADÃO, L. F. R. Produtividade e emprego: questões econômicas e estatísticas. Boletim de Conjuntura, IPEA, n. 31, p. 35-7, out. 1995.

COUTINHO, L. A especialização regressiva: um balanço do desempenho industrial pós-estabilização. In: VELLOSO, J. P. R. (Coord). Brasil: desafios de um país em transformação. Rio de Janeiro: José Olympio, 1997. p. 84-115.

ENDERS, W. Applied Economics Time Series. Iowa State Univversity. John \& Sons, 1995.

FEIJÓ, C. A. Produtividade do trabalho e emprego: o duplo desafio para os próximos anos. In: SICSÚ, J.; PAULA, L. F.; OREIRO, J. L. DA C. Agenda Brasil: políticas econômicas para crescimento com estabilidade de preços. São Paulo: Manole, 2003. p. 201-40.

FEIJÓ, C. A.; CARVALHO, P. G. M. Produtividade industrial no Brasil: o debate recente e as fontes de dados. In: ENCONTRO NACIONAL DE ESTUDOS DO TRABALHO, 6, Abet, 1999. Disponível em <http://www.race.nuca.ie.ufrj.br/abet/vienc/ST16A.doc >. Acesso em: 16 jul. 2003.

Recuperação econômica brasileira e outras questões recentes. Indicadores Econômicos FEE, Porto Alegre, v. 28, n. 3, p. 232-55, dez. 2000.

Sete teses equivocadas sobre o aumento da produtividade industrial nos anos recentes. Boletim de Conjuntura, Rio de Janeiro, IEI/UFRJ, p. 109-121, jul. 1994.

FRANCO, G. H. B. A inserção externa e o desenvolvimento. Revista de Economia Política, São Paulo, v. 18. n. 3-71, jul./set. 1998.

As "Reformas" e o crescimento da produtividade: uma nova economia. In: FONSTES, R.; ARBEX, M. A. Economia aberta: ensaios sobre fluxos de capitais, câmbio e exportações. Viçosa: UFV, 2000. p. 27-34. 
GOLDBERG, L.; TRACY, J. Exchange Rates and Local Labor Markets. NBER Working Paper, n. $6.985,1999$.

; AARONSON, S. Exchang rates and employment instability: evidenc from matched CPS data. The American Economic Review, Nova York, v. 89, n. 2, p. 204-09, maio 1999.

JOHANSEN, S. Likelihood-based inference in cointegrated vector auto-regressive models. (Advanced Texts in Econometrics). Nova York: Oxford University Press, 1995. 267 p.

MCCOMBIE, J. S. L.; THIRLWALL, A. P. Economic growth and balance of payments constraint. Nova York: St. Martin's Press, 1994.

PRIESTLEY, M. B. Spectral analyses and time series. Londres: Academic Pres, 1981.

RAMOS, L.; REIS, J. G. A. Emprego no Brasil: os anos 90. In: VELLOSO, J. P. dos R. (Coord.). Brasil: desafios de um país em transformação. Rio de Janeiro: José Olympio, 1997. p. 219-46.

REVENGA, A. Exporting jobs? The impact of import competition on employment and wages in us manufacturing. Quarterly Journal of Economics, n. 107 (1), p. 255-284, 1992.

RIVERA-BATZ, L. F.; RIVERA-BATIZ, L. A International Finance and Open Economy Macroeconomics. Nova Jersey: Prentice Hall, 1994.

ROCHA, F. Produtividade e mudança estrutural na indústria extrativa e de transformação brasileira, 1970-2001. Revista de Economia Política, v. 27, n. 2, 2007.

ROMER, D. Advanced Macroeconomics. Nova York: McGraw-Hill Companie, 1996.

ROSSI JÚNIOR, J. L.; FERREIRA, P. C. A evolução da produtividade industrial brasileira e abertura comercial. Pesquisa e Planejamento Econômico, Rio de Janeiro, v. 29, n.1, p. 1-35, abr. 1999.

SABOIA, J.; CARVALHO, P. G. M. de. Produtividade na indústria brasileira: questões metodológicas e análise empírica. Texto para Discussão, Brasília: IPEA, n. 504, ago. 1997.

SALM, C.; SABOIA, J.; CARVALHO, P. G. M. Produtividade na indústria brasileira: questões metodológicas e novas evidências empíricas. Pesquisa e Planejamento Econômico, Rio de Janeiro: IPEA, v. 27, n. 2, ago. 1997.

—; Produtividade na indústria brasileira: uma contribuição ao debate. In: CARLEIAL, L.; VALLE, R. (Org.). Reestruturação produtiva e mercado de trabalho no Brasil. São Paulo: Hucitec-Abet, 1997. p. 35-54.

SILVA, A. et al. Retrospectiva da economia brasileira. Perspectivas da Economia Brasileira, Rio de Janeiro: IPEA, 1994.

SIMONSEN, M. H.; CYSNE, R. P. Macroeconomia, 2. ed. Rio de Janeiro: FGV, 1995.

SOUZA NETO, C. R.; CURADO, M. L. Produtividade do trabalho, salários reais e desemprego na indústria de transformação na década de 90: teoria e evidência. Revista de Economia Contemporânea, Rio de Janeiro, v. 9, n. 3, p. 485-508, 2005.

STOCK, J. H.; WATSON, M. W. Vector autoregressions. Journal of Economic Perspectives, v. 15, n. 4, p. 101-115, 2001.

VILLELA, A.; SILVA, R. Ganhos de produtividade: aspectos conceituais, implicações econômicas. Revista do BNDES, Rio de Janeiro, v. 1, n. 2, p. 77-97, dez. 1994. 